\title{
En el artículo:
}

Características asociadas a la consulta pre viaje en turistas que visitan Cusco, Perú.

Christian R. Mejia, Aleksandar Cvetkovic-Vega, Briggite Cruz, Matlin M. Cárdenas,

Dante M. Quiñones-Laveriano y Alfonso J. Rodríguez-Morales

Rev Chilena Infectol 2016; 33 (1): 38-42

\section{El listado de autores dice:}

"Briggite Cruz"

Debe decir:

"Briggitte Cruz"

\section{En el artículo:}

Consenso chileno de prevención, diagnóstico y tratamiento de la diarrea asociada a Clostridium difficile

Rev Chilena Infectol 2016; 33 (1): 98-118

\section{El listado de autores dice:}

Cristian Hernández-Rocha, Paola Pidal, M. Cristina Ajenjo, Rodrigo Quera, Marcela Quintanilla, Jaime Lubascher, M. Irene Jemenao, Patricio Ibáñez, Manuel Álvarez-Lobos, Alexis Diomedi, Alejandra Marcotti, Mirta Acuña, Juan P. Arab, Arnoldo Riquelme, Roberto Candia y Sergio Carvajal

\section{Debe decir:}

Cristian Hernández-Rocha, Paola Pidal, M. Cristina Ajenjo, Rodrigo Quera, Marcela Quintanilla, Jaime Lubascher, M. Irene Jemenao, Patricio Ibáñez, Manuel Álvarez-Lobos, Alexis Diomedi, Alejandra Marcotti, Ismael Correa, Mirta Acuña, Juan P. Arab, Arnoldo Riquelme, Roberto Candia y Sergio Carvajal

\section{En el artículo:}

Consenso chileno de prevención, diagnóstico y tratamiento de la diarrea asociada a Clostridium difficile Rev Chilena Infectol 2016; 33 (1): 98-118

En la Presentación dice:

Revista Chilena de Gastroenterología

Debe decir:

Revista Gastroenterología Latinoamericana 